\title{
Development of the Selection and Manipulation of Self-Generated Thoughts in Adolescence
}

\author{
Iroise Dumontheil, ${ }^{1}$ Bano Hassan, ${ }^{2}$ Sam J. Gilbert, ${ }^{1}$ and Sarah-Jayne Blakemore ${ }^{1}$ \\ ${ }^{1}$ Institute of Cognitive Neuroscience, University College London, London WC1N 3AR, United Kingdom, and ²Great Ormond Street Children's Hospital, \\ London WC1N 3JH, United Kingdom
}

The ability to select and manipulate self-generated (stimulus-independent, SI), as opposed to stimulus-oriented (SO), information, in a controlled and flexible way has previously only been studied in adults. This ability is thought to rely in part on the rostrolateral prefrontal cortex (RLPFC), which continues to mature anatomically during adolescence. We investigated (1) the development of this ability behaviorally, (2) the associated functional brain development, and (3) the link between functional and structural maturation. Participants classified according to their shape letters either presented visually (SO phases) or that they generated in their head by continuing the alphabet sequence (SI phases). SI phases were performed in the presence or absence of distracting letters. A total of 179 participants (7-27 years old) took part in a behavioral study. Resistance to visual distractors exhibited small improvements with age. SI thoughts manipulation and switching between SI and SO thoughts showed steeper performance improvements extending into late adolescence. Thirtyseven participants (11-30 years old) took part in a functional MRI (fMRI) study. SI thought manipulation and switching between S0 and SI thought were each associated with brain regions consistently recruited across age. A single frontal brain region in each contrast exhibited decreased activity with age: left inferior frontal gyrus/anterior insula for SI thought manipulation, and right superior RLPFC for switching between SO and SI thoughts. By integrating structural and functional data, we demonstrated that the observed functional changes with age were not purely consequences of structural maturation and thus may reflect the maturation of neurocognitive strategies.

\section{Introduction}

Adolescence is a time of profound mental change, affecting many aspects of behavior including planning, multitasking and the ability to resist distractions (Blakemore and Choudhury, 2006). These abilities recruit "executive functions," which enable the selection of actions that are consistent with one's goal. While most aspects of executive functions are well established by late childhood, the ability to deploy them flexibly and consistently continues to develop during adolescence (Luna et al., 2010). An important aspect of executive function in adults is the ability to modulate the balance between cognition that is provoked by perceptual experience (stimulus-oriented, $\mathrm{SO}$ ) and that which occurs in the absence of sensory input (stimulus-independent, SI) (Burgess et al., 2007). No previous study has directly investigated the development of this ability. In children, manipulation of SI thoughts has been studied in the context of fluid intelligence (Wright et al., 2008; Crone et al., 2009) and working memory (WM) tasks (Crone et al., 2006), while the ability to resist distracting SO information has been studied in perceptual (Bunge et al., 2002; Booth et al., 2003) and WM tasks (Olesen et al., 2007). Here, we used a single task that could be performed on the basis of either SO or SI information, without high WM requirements

Received March 17, 2010; accepted April 19, 2010.

This research was supported by grants from the Royal Society to S.J.B. and S.J.G. and a Fellowship from the European Commission to I.D. We are grateful to A. Ranpura for his help with the VBM analyses.

Correspondence should be addressed to Dr. Iroise Dumontheil, UCL Institute of Cognitive Neuroscience, 17 Queen Square, London WC1N 3AR, UK. E-mail: i.dumontheil@ucl.ac.uk.

DOI:10.1523/JNEUROSCI.1375-10.2010

Copyright $\odot 2010$ the authors $\quad 0270-6474 / 10 / 307664-08 \$ 15.00 / 0$
(Gilbert et al., 2005, 2007, 2008). Distracting SO information was present or absent during SI phases of the task.

The studies mentioned above report observations during adolescence of improvements in WM and fluid reasoning performance, and to a lesser extent of the resistance to perceptual distractors, as well as changes in neural activity, in particular in the prefrontal cortex (PFC). Decreases are frequently interpreted as being due to developmental reductions in gray matter volume, presumably related to synaptic pruning, while increases are thought to relate to improved and more localized task-specific processing, potentially facilitated by faster long-range connections due to increased axonal myelination and size (Luna et al., 2010). Understanding the link between structural and functional changes is critical in understanding the mechanisms of neurocognitive development, yet very few studies have directly compared structural and functional data within the same individuals (Olesen et al., 2003; Lu et al., 2009).

An a priori region of interest was the rostrolateral prefrontal cortex (RLPFC), which has been suggested to support the selection and manipulation of SI thoughts, as opposed to SO information, in a controlled and flexible way (Christoff and Gabrieli, 2000; Burgess et al., 2005; Gilbert et al., 2005; Dumontheil et al., 2008). In a behavioral study and a functional magnetic resonance imaging (fMRI) study, we investigated: (1) how performance on a task requiring selection and manipulation of SI thoughts develops between late childhood and adulthood, differentiating between three aspects of the task: selection and manipulation of SI versus SO thoughts; switching versus maintaining the selection of SI or SO thoughts; and resistance to distractors; (2) how neural 


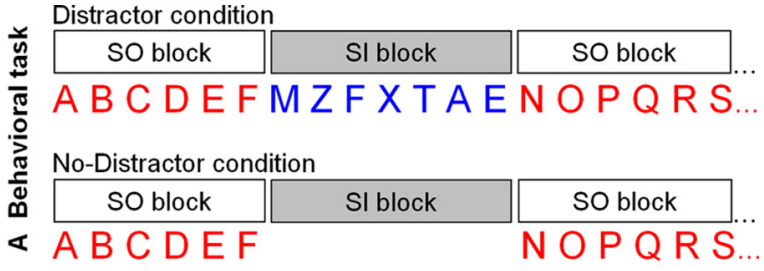

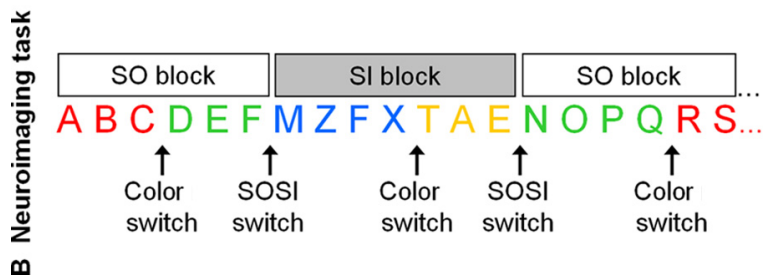

Figure 1. Examples of sequences of trials from the alphabet task used in the behavioral and neuroimaging studies. $A$, In the behavioral task, participants were required to classify letters as consisting of straight lines only or containing a curve. In $\mathrm{SO}$ phases, participants classified the red letter presented on the screen. In SI phases, participants classified the letter in their head, generated by continuing the alphabet sequence. During SI phases either a random blue letter was presented on the screen (Distractor condition) or the screen was blank (No-Distractor condition). Switch trials were the first trial after a change between SI/SO phases; stay trials were defined as all subsequent trials. $\boldsymbol{B}$, The neuroimaging task included the Distractor condition only. Letters were red or green in SO blocks, blue or yellow in SI blocks. Participants were trained to learn this association. A change in color of the letters within $\mathrm{SO}$ or $\mathrm{SI}$ blocks was coded as a Color switch, while a change in color of the letters between $\mathrm{SO}$ and SI blocks was coded as an SOSI switch.

activity underlying these processes develops between early adolescence and adulthood; and (3) whether age-related changes could be accounted for by changes in gray and white matter volumes.

\section{Materials and Methods}

Both studies were restricted to female participants so as to reduce variance attributable to sex differences in brain maturation and cognitive development (Giedd et al., 1999, 2006). Written informed consent was obtained before both studies from all participants, or from the parent of participants aged under 18. The studies were approved by the University College London (UCL) National Hospital for Neurology and Neurosurgery Ethics Committee. Stimulus presentation was programmed in Cogent (www.vislab.ucl.ac.uk/Cogent/index.html) running in Matlab 6.5 (MathWorks), which recorded participants' responses.

\section{Behavioral study}

Participants. A total of 179 female volunteers between the ages of 7.3 and 27.5 years were recruited for this study. Children and adolescents $(n=143)$ were recruited from two London schools for girls, while adults $(n=36)$ were recruited from the UCL Psychology Department volunteer database. All participants spoke English as their first language. Verbal ability was measured in children and adolescents using the BPVS II scores (Dunn et al., 1997), and in adults using the Vocabulary subtest of the WASI (Wechsler, 1999). Data from one adolescent who had a verbal intelligence quotient (IQ) score of $<75$ were excluded from the analysis. There was no significant correlation between verbal IQ and age (Pearson $r=0.008, p>0.8$ ).

Design. The behavioral task had three within-subjects factors (Condition: Distractor or No-Distractor; Block type: SO or SI; and Trial type: Switch or Stay), and one between-subjects factor (Age). We used a computer-based task adapted from Gilbert et al. (2005), which tests the control of the allocation of attention between SO and SI information (Fig. 1). In SO blocks a single upper case letter was presented in the center of the screen in red. Participants were required to classify the letter according to its shape by pressing one of two "Ctrl" buttons on a laptop keyboard, using their left and right index fingers. Participants were asked to press the left key if the letter was composed entirely of straight lines (e.g., the letter A) or the right key if the letter shape contained a curve (e.g., the letter B). After each button press a new letter was presented, following the sequence of the alphabet. During SI blocks, participants were asked to continue to go through the alphabet sequence and to continually classify as curved/straight-lined each of the letters in their head and press the corresponding keys. In the No-Distractor condition, the screen during SI blocks was blank. In the Distractor condition, a blue letter, pseudo-randomly picked from the alphabet with the constraint that it was different from the current and previous letters the participant had in her head, was shown in the middle of the screen, with a new letter after each button press. Participants were instructed to ignore this letter. Eventually a new SO phase began and the red letters reappeared continuing the alphabet sequence. The Distractor condition enabled us to explore the proposal that selection between SI and SO thoughts is particularly important when it does not occur naturally or automatically (as in the absence of competing SI or SO information) but is instead imposed in a controlled way (when both SI and SO information are present) (Burgess et al., 2005). Analyses differentiated between: (1) performance on the first trial of SO and SI blocks, i.e., "switch" trials, which indicates participants' ability to switch between selecting SO and SI information; and (2) performance on the subsequent trials of SO and SI blocks, i.e., "stay" trials, which indicates participants' ability to maintain the selection and processing of SO or SI information.

SO and SI phases alternated and lasted on average 6.5 trials (range 5-8 trials). Switches between SO and SI phases were cued by the appearance of a letter (red letter: SO; blue letter or blank: SI). Participants performed one run of the No-Distractor condition and one run of the Distractor condition; order was counterbalanced between participants. Each run comprised six SO and six SI phases. All conditions were self-paced with no interval between the displays of stimuli.

Instructions for the task were displayed on the screen and read out loud by the experimenter. Participants first performed for practice the SO task for two repetitions of the alphabet sequence ( 52 trials). Relevant additional instructions regarding SI phases and a run of 26 practice trials with alternating SO and SI phases preceded the runs of the Distractor and No-Distractor condition.

Participants were tested individually in a quiet room at their school or at home (for the children and adolescents) or in the laboratory (for the adults).

Data analysis. Mean accuracy and median response times (RT) in correctly responded trials were calculated for each participant in each condition. Main effects were investigated using paired $t$ tests. Changes in performance with age for accuracy and RT overall and for the effects of each factor separately were investigated using regression analyses. Linear, quadratic and cubic age regressors were added in turn to the model until further improvement to the model was not significant. Bayesian information criterion (BIC), or Schwartz criterion, was calculated and in all cases confirmed the final model selection was not driven by increased model complexity only (Schwartz, 1978; Liddle, 2007). The models were subsequently used to calculate for each factor the amplitude of accuracy or RT changes between the youngest age and the age at the first inflection of the fit curve (or the oldest age for linear fits).

\section{Neuroimaging study}

Participants. Thirty-seven right-handed, female participants aged between 11.0 and 30.4 years, with no history of psychiatric or neurological disorder, took part in the study. Adolescents $(n=24)$ were recruited through advertisements at their schools or following their parents' response to an article in a newspaper. Adults $(n=13)$ were recruited following their participation via an advert in a local community electronic newsletter or in the department. General ability of the participants was measured using the Vocabulary and Matrix Reasoning subtests of the WASI (Wechsler, 1999). There was no significant correlation between IQ and age (Pearson correlation $r=0.028, p>0.8$ ).

Experimental design. The fMRI task was a further adaptation of the alphabet task (Gilbert et al., 2005). The task had two factors: Block type (SO, SI) and Trial type (Stay, Color switch, SOSI switch). In all trials a single upper case letter was presented on the screen, in one of four possible colors (Fig. 1). Two colors were associated with SO blocks (e.g., red and green) and two colors with SI blocks (e.g., blue and yellow). Color 
switch trials were included as a control condition in which the stimulus color changed (as in SOSI switch trials) but the Block type (SO, SI) did not. Instructions for the SO and SI conditions were the same as in the Distractor condition of the behavioral study (see above). Participants used their right index and middle fingers to respond.

The task was self-paced with no interval between the presentations of successive letters. SO and SI phases alternated: SO phases lasted $10.5 \mathrm{~s}$ on average (average range $3.4-15.5 \mathrm{~s}$ ); SI phases lasted $9.3 \mathrm{~s}$ on average (average range $2.8-16.5 \mathrm{~s}$ ). Color switches occurred on average $5.3 \mathrm{~s}$ after the start of an SO (average range 2.1-7.5 s) or SI block (average range $2.6-8.1 \mathrm{~s}$ ). Task blocks and fixation blocks alternated, and lasted on average $40.5 \mathrm{~s}$ (average range $40.0-45.3 \mathrm{~s}$ ) and $14.7 \mathrm{~s}$ (average range 13.6-15.0 s), respectively. There were two scanning sessions, each comprising eight task blocks and seven fixation blocks.

Before the scanning participants performed supervised practice of the tasks, which was continued until participants performed at least 20 correct responses in a row, after a minimum of three blocks of the task and an initial practice of two sets of $26 \mathrm{SO}$ trials.

MRI data acquisition. A 1.5 Tesla Siemens Avanto MRI scanner was used to acquire both $3 \mathrm{D} \mathrm{T}_{1}$-weighted fast-field echo structural images and multislice $\mathrm{T}_{2}{ }^{*}$-weighted echo-planar volumes with blood oxygen level-dependent $(\mathrm{BOLD})$ contrast $(\mathrm{TR}=3 \mathrm{~s} ; \mathrm{TE}=50 \mathrm{~ms} ; \mathrm{TA}=2.9143$ s). For each subject, functional data were acquired in two scanning sessions of the alphabet task of $7.45 \mathrm{~min}$ duration. This was preceded by two 7 min scanning sessions of a separate task and acquisition of the structural images. The first 2 volumes of each session were discarded to allow for $\mathrm{T}_{1}$ equilibrium effects. Each functional brain volume comprised 35 axial slices with a resolution of $3 \times 3 \times 3 \mathrm{~mm}$, positioned to cover the whole brain.

Statistical analysis. Mean of the participants' median RTs and percentage accuracy were calculated. Main effects were investigated using paired $t$ tests. Changes in performance with age in overall accuracy, overall RT, and in the effects of interest (SI stay vs SO stay; SOSI switch vs Color switch) were investigated using regression analyses. Linear, quadratic and cubic age regressors were added in turn to the model until further improvement to the model was not significant.

fMRI data were analyzed using statistical parametric mapping implemented in SPM5 (http://www.fil.ion.ucl.ac.uk/spm). Volumes were realigned (using fourth-degree-B-spline interpolation), corrected for differences in slice acquisition times, normalized into $3 \mathrm{~mm}$ cubic voxels using the subjects' structural $(n=35)$ or mean functional scans $(n=2$, subjects with lower quality structural scans) to the standard T1 or EPI template based on the Montreal Neurological Institute (MNI) reference brain, respectively, and spatially smoothed with an isotropic $8 \mathrm{~mm}$, fullwidth, half-maximum Gaussian kernel.

Analysis of the movement parameters obtained during realignment showed that translation within each session was $<3 \mathrm{~mm}$ in all participants except in the second session of one participant (translation in $z$ plane $>8 \mathrm{~mm}$ ). This session was excluded from all analyses. Correlation analyses performed on the participants' means across sessions showed a significant linear decrease in rotation in the $y$-axis with age (Pearson $r=$ $-0.365, p=0.026$ ). The five other movement parameters did not show significant correlations with age (all $p>0.1$ ).

The volumes acquired during the two sessions were treated as separate time series. For each series, the variance in the BOLD signal was decomposed with a set of regressors in a general linear model (Friston et al., 1995). The regressors were four boxcar regressors representing the instructions, fixation, SO and SI blocks, and three event-related regressors representing color switches, SOSI switches, and error trials. All regressors were convolved with a canonical hemodynamic response function and, together with regressors representing residual movement-related artifacts and the mean over scans, comprised the full model for each session. The data and model were high-pass filtered to a cutoff of $1 / 128 \mathrm{~Hz}$.

Parameter estimates calculated from the least mean squares fit of the model to the data were used in a one sample $t$ test (color switch, SOSI switch) or pairwise contrasts (SO $>$ fixation, $\mathrm{SI}>$ fixation) at the individual subject level. The participants were divided into three groups of similar $n$ (age $11-14, n=12$; age $14-18, n=12$; age 22-30, $n=13$ ). First level contrasts were entered into two separate 2 (Condition) $\times 3$ (age group) factorial design second level analyses, where 'subject' was treated as a random effect. The within-subjects factors were block type (SO vs SI) and switch type (color switch vs SOSI switch) respectively, and age group was the between-subjects factor. These analyses had two aims: first, to study any effect of age group as a categorical variable; second, to obtain a main effect of condition unbiased by age to define regions of interests in which to test for effects of age as a continuous variable on neural activity and on local gray and white matter volumes. Main effects of block type and switch type and their interaction with age were analyzed using weighted linear contrasts and determined using the $t$-statistic on a voxel by voxel basis. Tests were corrected for family-wise errors (FWE) across the whole brain, and in our region of interest (ROI) RLPFC, using an anatomically defined Brodmann area 10 (BA10) mask from WFU PickAtlas Tool Version 2.4 (Maldjian et al., 2003, 2004). Parameter estimates of the peak voxel of clusters showing an effect of experimental condition were extracted and analyzed for effects of age using Pearson tests of correlations. It should be noted that this is an unbiased method as the voxels of interest were defined from a fully balanced contrast orthogonal to the effect of age (Kriegeskorte et al., 2009). In a second step, multiple regressions were performed on the peak voxels showing correlation between BOLD signal and age to test whether age still accounted for changes in BOLD signal when individual structural and performance differences were taken into account.

Voxel-based morphometry. Voxel-based morphometry (VBM) (Ashburner and Friston, 2000) investigating gray and white matter tissue volumes was performed on all participants using SPM5 VBM5 toolbox (v1.15 http://dbm.neuro.uni-jena.de/vbm/). Each participant's structural T1 image was normalized to the standard T1 MNI template. Segmentation was performed without using prior tissue probability maps, which is the option favored when testing children and adolescents. Structural scans were segmented into CSF, gray and white matter; modulation for nonlinear warping only was performed using the Jacobian determinants. This method approximates a proportional adjustment of volumes for overall head size (O'Brien et al., 2006). Images were resampled into $1.5 \times$ $1.5 \times 1.5$ voxels and smoothed with an isotropic $12 \mathrm{~mm}$, full-width, half-maximum Gaussian kernel. The MarsBaR toolbox for SPM5 (http:// marsbar.sourceforge.net/) was used to calculate mean gray and white matter adjusted volumes within spheres of $5 \mathrm{~mm}$ in radius, centered around the peak voxels of the main contrasts that exhibited significant age effects. Gray and white matter adjusted volumes were investigated for effects of age using Pearson tests of correlation.

\section{Results}

\section{Behavioral study}

We instructed 178 participants aged 7.3-27.5 years to classify according to their shape letters that were either presented on the screen (SO) or not (SI), and in the presence or absence of distractors (Fig. 1). Age-related changes in overall performance and in the effect of each factor (Distractor-No-Distractor, SI-SO, and Switch-Stay) were investigated separately for accuracy and response time (RT) using regression analyses. Linear, quadratic and cubic age regressors were added in turn to the model until further improvement to the model was not significant.

Participants were slower and made more errors in the Distractor condition [mean (SD) accuracy and RT: Distractor: $93.8 \%$ (7.3), 1106 ms (372); No-Distractor: 95.0\% (5.0), 986 ms (324)], SI blocks [(SI 93.9\% (6.8), 1142 ms (430); SO: 94.9\% (5.2), 951 $\mathrm{ms}(273)$ ], and Switch trials [Switch: $94.1 \%$ (6.4), $1270 \mathrm{~ms}$ (466); Stay: $94.8 \%$ (4.6), $822 \mathrm{~ms}(228)]$ than in their respective control conditions (accuracy: all $t_{177}>1.99, p<0.05$; RT: all $t_{177}>8.38$, $p<0.001)$. Note that the presence of distractors in SI phases affected both SO and SI RTs (all $t_{177}>5.78, p<0.001$ ) and SO accuracy in the Distractor condition (SO: $t_{177}=2.69, p=0.008$; SI: $t_{177}<1$ ) [No-Distractor SO: $95.7 \%$ (4.9), 910 ms (272); Dis- 

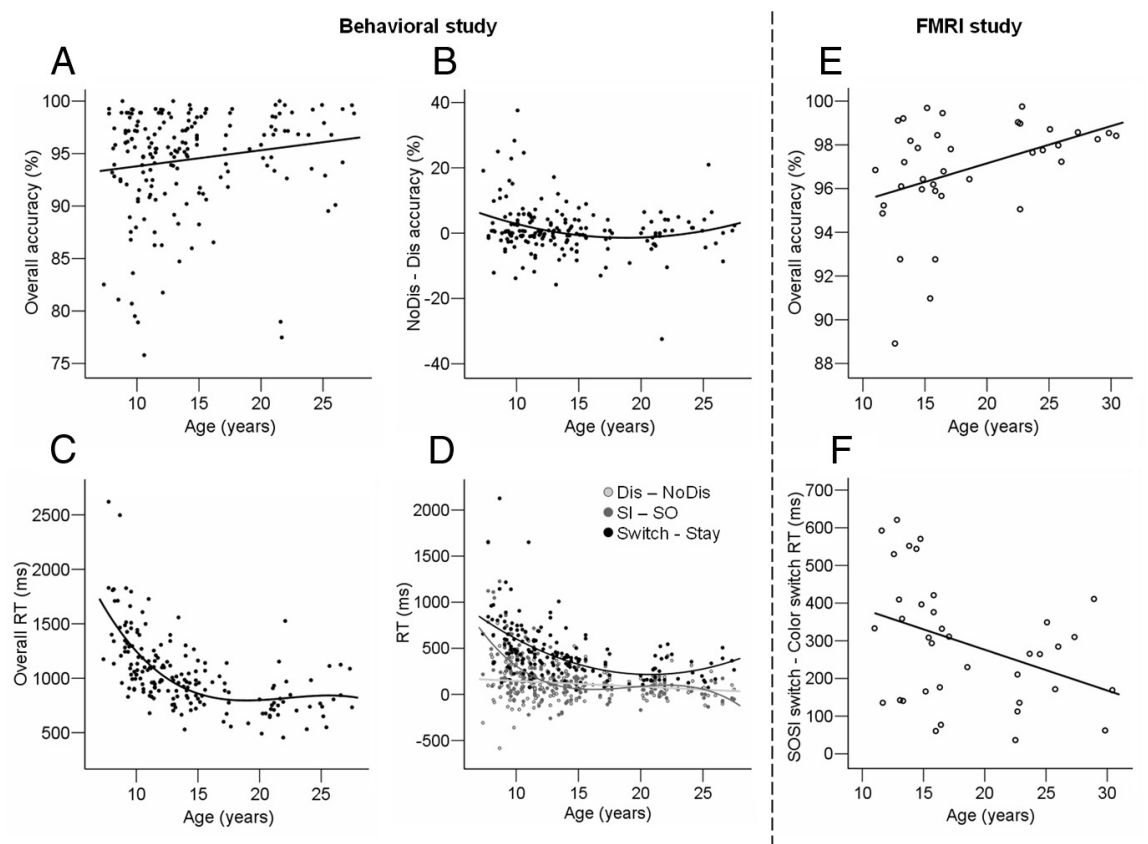

Figure 2. Accuracy and RT data from the behavioral study and fMRI study. Regression analyses were performed to test for linear, quadratic or cubic changes in performance with age. Significant effects observed in the behavioral study $(\boldsymbol{A}-\boldsymbol{D})$ or in the fMRI study $(\boldsymbol{E}, \boldsymbol{F})$ are shown in this figure. $\boldsymbol{A}$, Scatterplot of the overall accuracy (percentage) against age. The line represents the significant linear fit of the data. $\boldsymbol{B}$, Scatterplot of the difference in accuracy between No-Distractor and Distractor conditions against age. The curve represents the significant quadratic fit of the data. C, Scatterplot of the overall RTs (in milliseconds) against age. The curve represents the significant cubic fit of the data. $\boldsymbol{D}$, Scatterplot of the three main effects of the task on RT (in milliseconds) against age: in light gray, difference between Distractor and No-Distractor, with a significant linear fit; in dark gray, difference between SI and SO blocks, with a significant cubic fit; in black, switch cost (SOSI Switch-Stay), with a significant quadratic fit. $E$, Scatterplot of the overall accuracy (percentage) against age. The line represents the significant linear fit of the data. $\boldsymbol{F}$, Scatterplot of the RT switch cost (SOSI switch-Color switch) against age. The line represents the significant linear fit of the data.
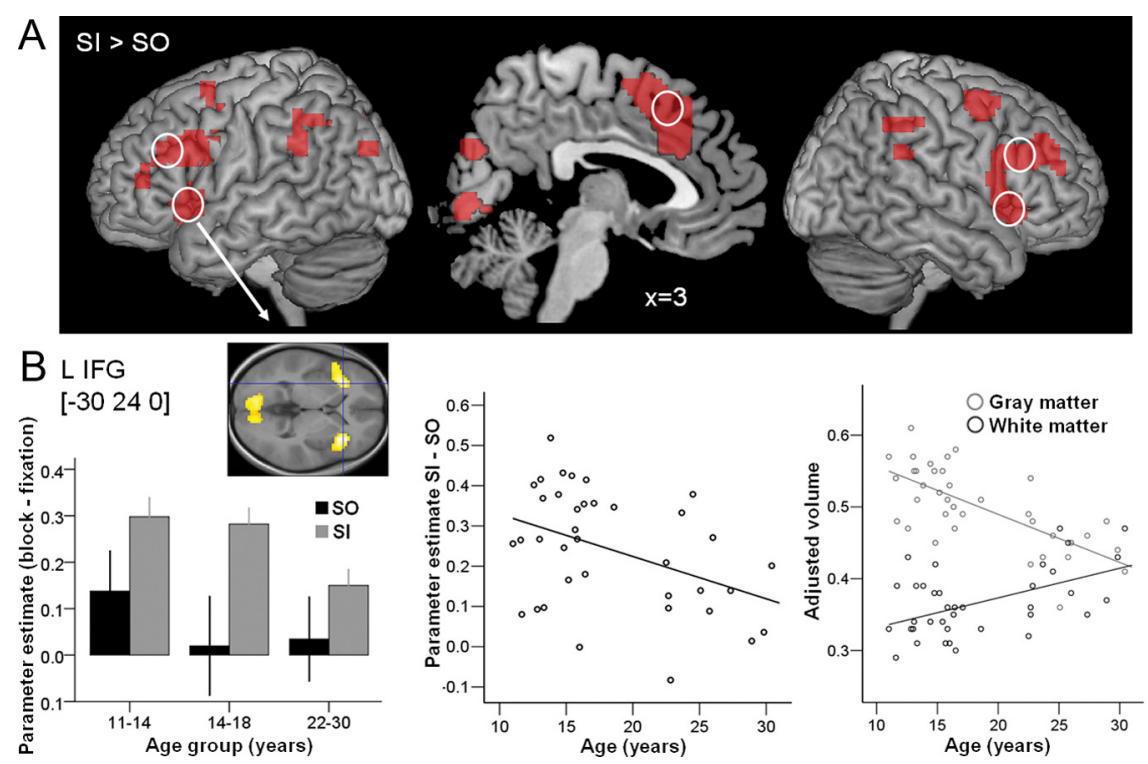

Figure 3. Neuroimaging results of the main effect $\mathrm{SI}-\mathrm{SO}$. $A$, Results of the $\mathrm{SI}-\mathrm{SO}$ contrast averaged across the three age groups are rendered on the brain surface (outer panels) and shown on the sagittal slice $(x=3)$ (middle panel) (FWE, $p<0.05$ ). The white circles indicate the approximate location of the regions in which the parameter estimates were extracted in the peak voxel and gray and white matter volumes were calculated in $5 \mathrm{~mm}$ spheres around the peak. $\boldsymbol{B}$, Changes in brain structure and function with age are presented for the only region showing an effect of age on the SI-SO contrast, the left IFG. Left, Bar chart representing IFG activation in $\mathrm{SI}$ and $\mathrm{SO}$ blocks vs fixation blocks in the three age groups (mean $\pm \mathrm{SE}$ ). Middle, Scatterplot of the BOLD response difference between $\mathrm{SI}$ and $\mathrm{SO}$ blocks as a function of age. Right, Scatterplot of gray (gray circles) and white (black circles) matter volume as a function of age. The full lines represent significant correlations between functional or structural measures and age. tractor SO: 94.1\% (7.8), $991 \mathrm{~ms}(304)$; No-Distractor SI: 94.3\% (7.8), $1062 \mathrm{~ms}$ (424); Distractor SI: 93.5\% (9.8), $1221 \mathrm{~ms}$ (480)].

Average accuracy was $>90 \%$ in all conditions. Regression analyses indicated that overall accuracy improved linearly with age $\left(F_{(1,176)}=4.44, p=0.037, \beta_{\text {age }}=\right.$ $0.16, R^{2}=0.02$ ) (Fig. $2 A$ ). Of the three main effects, only Condition (Distractor vs No-Distractor) was associated with age, with the difference in accuracy between Distractor and No-Distractor blocks following a quadratic function $\left(F_{(2,175)}=5.72\right.$, $p=0.004, \beta_{\text {age }}=-1.39, \beta_{\text {age }^{2}}=1.24$, $R^{2}=0.06$ ) (Fig. $2 B$ ). This reflected an improvement with age in accuracy in the Distractor $(p=0.010)$ but not the NoDistractor condition $(p>0.29)$. The inflection of the quadratic curve occurred at age 18.8 years, suggesting that the accuracy cost of the presence of Distractors reached adult levels by this age.

Overall RT decreased with age following a cubic function $\left(F_{(3,174)}=48.49, p<0.001\right.$, $\beta_{\text {age }}=-6.52, \beta_{\text {age }^{2}}=9.95, \beta_{\text {age }^{3}}=-4.03$, $R^{2}=0.46$ ) (Fig. 2C). The first inflection of this cubic curve occurred at age 18.9 years. All main effects showed changes with age, with decreases in the RT difference between Distractor and No-Distractor (linear model: $F_{(1,176)}=5.16, p=0.024, \beta_{\text {age }}=-0.17$, $R^{2}=0.03$ ), SI and SO (cubic model: $F_{(3,174)}=$ 27.96, $p<0.001, \beta_{\text {age }}=-9.17, \beta_{\text {age }^{2}}=$ $\left.16.48, \beta_{\text {age }^{3}}=-7.86, R^{2}=0.33\right)$ and Switch and Stay trials (quadratic model: $F_{(2,175)}=$ $40.25, p<0.001, \beta_{\text {age }}=-2.40, \beta_{\text {age }^{2}}=1.94$, $R^{2}=0.32$ ) (Fig. 2D). Inflections of the estimated functions occurred at 16.2 years for the SI-SO difference, and at 20.7 years for the Switch-Stay difference. The difference between [Switch-Stay] and [SI-SO] did not show any significant change with age $(p>$ $0.15)$, however, which suggests that the time courses of the two effects did not differ significantly.

\section{Neuroimaging study}

Behavioral results

In the fMRI study, 37 participants aged 11.0-30.4 years were scanned while carrying out an adapted version of the alphabet task in which there was no No-Distractor condition (Fig. 1). Changes in performance with age in overall accuracy and RT, and in the differences in accuracy and RT between SI and SO stay trials and between SOSI and Color switch trials, were investigated using regression analyses.

Average accuracy was $>90 \%$ in all conditions. Regression analyses indicated that overall accuracy increased linearly with age $\left(F_{(1,35)}=6.92, p=0.013, \beta_{\text {age }}=\right.$ 
$0.41, R^{2}=0.17$ ) (Fig. $2 E$ ). Participants made more errors in SI vs SO stay blocks $\left(t_{36}=2.38, p<0.05\right)$, but not in SOSI switch vs Color switch trials $(p>0.6)$. The differences in accuracy between SI and SO stay trials, and between SOSI switch and Color switch trials, did not show significant changes with age.

Participants were slower in SI vs SO stay blocks, and SOSI switch vs Color switch trials (both $t_{36}>4.99, p<0.001$ ). Overall $\mathrm{RT}$, and the RT difference between SI and SO stay trials, did not change with age. The RT switch cost (SOSI switch-Color switch) decreased linearly with age $\left(F_{(1,35)}=6.06, p=0.019, \beta_{\text {age }}=\right.$ $-0.38, R^{2}=0.15$ ) (Fig. $2 F$ ).

These fMRI behavioral results suggest later performance improvement for switching between SI and SO thoughts than for maintaining the selection of SI thoughts, while the developmental time course of these two factors did not differ in the behavioral study. The differences observed may arise from the narrower and older age range used in the fMRI study, and more extensive task practice in the fMRI study.

\section{fMRI results}

Main effects of condition. The effects of the two experimental factors were assessed using two 2 (condition) $\times 3$ (age group) factorial analyses (see Materials and Methods). [SI-SO] and [SOSI switch-Color switch] main effect contrasts were performed averaging across participants divided into three age groups [early adolescence: $11-14$ years old $(n=12)$; mid adolescence: $14-17$ years old $(n=12)$; adults $(n=13)]$. Resulting activations surviving whole brain or within BA10 FWE correction at $p<0.05$ are reported.

The SI-SO block comparison revealed bilateral activation of the inferior frontal gyri (BA46/44/45), extending into the insula (Fig. $3 B$, see bottom left plot), and of the premotor cortex, parietal cortex, medial frontal cortex, and the precuneus (Table 1, Fig. 3). Activations within our BA10 ROI were observed in left ([ -36 45 21], $Z=4.72$; [-39 48 12], $Z=4.69$; [-30 51 9], $Z=4.32$ ) and right RLPFC ([36 42 30], $Z=5.92$; [36 48 18], $Z=4.84$; [30 513 ], $Z=4.06)$.

The SOSI switch-Color switch comparison revealed greater activations in right superior RLPFC, cingulate gyrus (BA32), precuneus (BA7/18), cuneus (BA17) and superior temporal gyrus (BA22) (Table 1, Fig. 4). Activations within our BA10 ROI were observed in left ([ $\left.\left.\begin{array}{lll}-33 & 51 & 24\end{array}\right], Z=4.09\right)$ and right superior RLPFC ([36 4530$], Z=6.48$ ) (Fig. $4 B$, see bottom left plot).

Note there were no significant interactions between condition and age group or main effects of age group in these whole-brain analyses (FWE $p<0.05$ ), indicating that age group as a categorical variable did not affect neural activations in the SI-SO and SOSI switch-Color switch contrasts.

Effects of age on brain structure and function. Parameter estimates were extracted from the peak voxels of the main effect analyses and evaluated using orthogonal contrasts testing for significant changes in brain activation with age as a continuous variable. Defining voxels of interest based on functional data can be biased (Kriegeskorte et al., 2009). Here, the contrasts defining our voxels of interest were orthogonal to age. It should be noted that the results are reported without correction for multiples comparisons and therefore should be interpreted with caution and require further replication. VBM was used to extract average volumes of gray and white matter adjusted for head size in $5 \mathrm{~mm}$ spheres around the peak voxels exhibiting age-related effects to test for structural changes with age in these regions. Finally, multiple regression analyses were performed to test whether the observed age differences in BOLD signal reflect
Table 1. Whole-brain analyses (FWE, $p<0.05,10$ voxels or more) of the main effects of interest, SI-SO and SOSI switch-Color switch, averaged across the three age groups

\begin{tabular}{|c|c|c|c|c|}
\hline Label & $B A$ & $x, y, z$ (MNI) & $n$ & Z \\
\hline \multicolumn{5}{|l|}{ SI-SO } \\
\hline \multirow[t]{2}{*}{ Inferior frontal gyrus } & 47 & $-30,24,0$ & \multirow[t]{2}{*}{203} & 7.51 \\
\hline & 47 & $-51,15,0$ & & 6.15 \\
\hline Middle frontal gyrus & 46 & $-39,21,27$ & \multirow[t]{3}{*}{265} & 7.49 \\
\hline Inferior frontal gyrus & 44 & $-51,18,27$ & & 7.16 \\
\hline Inferior frontal gyrus & 9 & $-39,9,30$ & & 6.56 \\
\hline Superior frontal gyrus & 8 & $3,21,48$ & \multirow[t]{3}{*}{479} & 7.38 \\
\hline Cingulate gyrus & 32 & $9,21,33$ & & 6.55 \\
\hline Middle frontal gyrus & 6 & $36,0,57$ & & 6.47 \\
\hline Insula & 13 & $36,18,3$ & \multirow[t]{3}{*}{398} & 7.36 \\
\hline Middle frontal gyrus & 45 & $45,24,24$ & & 6.19 \\
\hline Inferior frontal gyrus & 44 & $51,12,12$ & & 5.95 \\
\hline \multirow[t]{2}{*}{ Lingual gyrus } & 18 & $18,-78,-9$ & \multirow[t]{3}{*}{470} & 6.66 \\
\hline & 18 & $-6,-78,0$ & & 6.58 \\
\hline Cuneus & 17 & $-9,-87,3$ & & 6.27 \\
\hline Precuneus & 7 & $-9,-72,42$ & 40 & 6.26 \\
\hline \multirow[t]{2}{*}{ Inferior parietal lobule } & 40 & $-60,-42,33$ & \multirow[t]{2}{*}{77} & 5.93 \\
\hline & 40 & $-39,-48,42$ & & 5.89 \\
\hline Lingual gyrus & 19 & $27,-54,-9$ & 17 & 5.61 \\
\hline Superior frontal gyrus & 6 & $-24,9,60$ & 22 & 5.54 \\
\hline Middle frontal gyrus & 9 & $-27,33,27$ & 12 & 5.33 \\
\hline Angular gyrus & 39 & $-36,-81,30$ & 12 & 5.33 \\
\hline \multirow[t]{2}{*}{ Inferior parietal lobule } & 40 & $57,-39,42$ & \multirow[t]{2}{*}{13} & 5.26 \\
\hline & 40 & $57,-30,45$ & & 4.98 \\
\hline \multicolumn{5}{|l|}{ SOSI switch-Color switch } \\
\hline Middle frontal gyrus & $10 / 9$ & $36,45,30$ & 25 & 6.48 \\
\hline \multirow[t]{3}{*}{ Precuneus } & 7 & $0,-57,54$ & \multirow[t]{3}{*}{149} & 6.19 \\
\hline & 7 & $9,-66,54$ & & 5.79 \\
\hline & 7 & $-9,-75,48$ & & 5.61 \\
\hline Precuneus & 18 & $0,-78,24$ & 15 & 5.43 \\
\hline \multirow[t]{3}{*}{ Cuneus } & 17 & $9,-81,6$ & \multirow[t]{3}{*}{28} & 5.23 \\
\hline & 17 & $0,-84,9$ & & 5.17 \\
\hline & 17 & $-6,-93,9$ & & 4.83 \\
\hline Superior temporal gyrus & 22 & $-57,-48,18$ & 13 & 5.1 \\
\hline
\end{tabular}

n, cluster size; Z, Z score.

differences in brain structure and/or performance (accuracy and RT).

SI-SO. No change in BOLD signal with age was observed in the RLPFC in the SI-SO contrast. However, the left IFG [ -30 24 0 ] (extending into the insula in the whole-brain analysis) exhibited a significant linear decrease in activation with age (Pearson $r=-0.417, p=0.010)$. VBM analyses revealed that gray matter volume in this region exhibited a significant decrease with age $(r=-0.695, p<0.001)$, while white matter volume in this region exhibited a significant increase with age $(r=-0.511, p=0.001)$ (Fig. 3B).

To investigate whether the correlation between age and BOLD signal was maintained when taking into account individual differences in structural development and in performance, a regression analysis was performed with gray and white matter adjusted volumes and differences in accuracy and RT between SI and SO stay trials as regressors. Results indicated that age still showed a trend toward accounting for BOLD response variance $(\beta=$ $-0.378, p=0.099$ ), while none of the structural or performance regressors showed significant effects (all $p>0.26$ ) (Table 2).

SOSI switch-color switch. Again, only one region observed in the whole-brain analysis of the main effect of SOSI switch-Color switch exhibited changes in BOLD signal with age. This was the right superior RLPFC region [36 4530 ], where the difference in BOLD signal between SOSI switch and Color switch marginally 


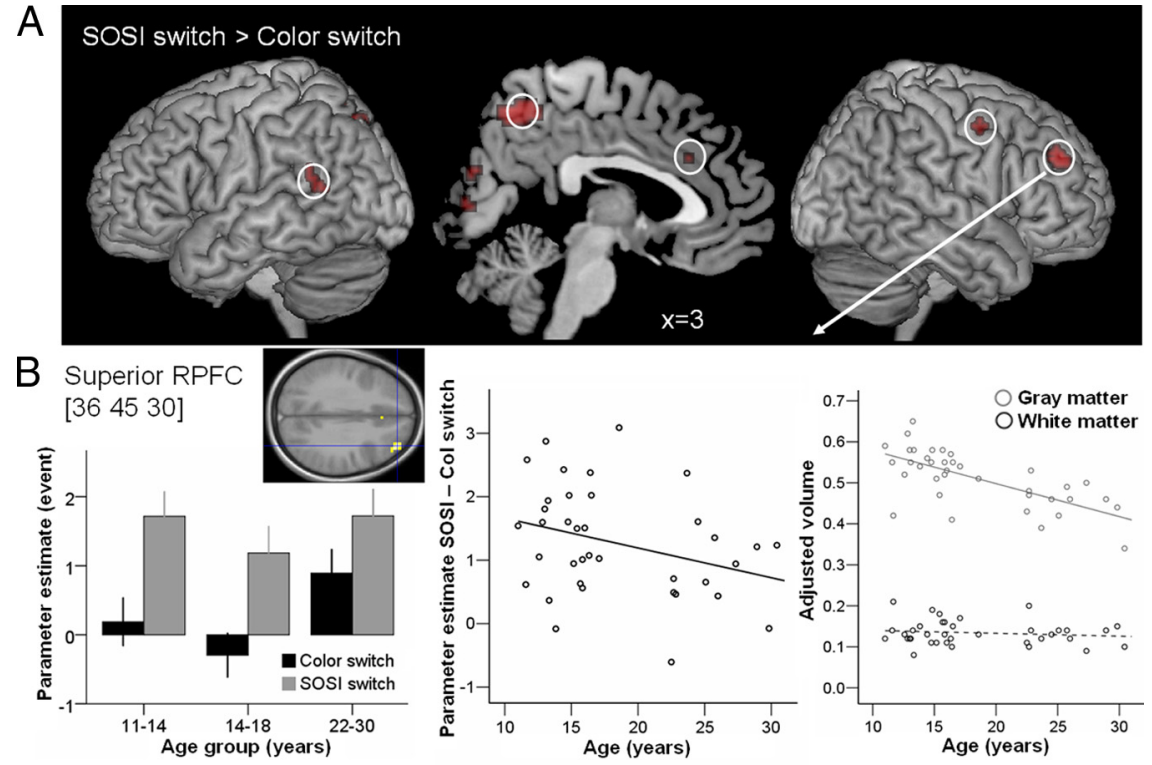

Figure 4. Neuroimaging results of the main effect SOSI switch-Color switch. $A$, Results of the SOSI switch-Color switch contrast averaged across the three age groups are rendered on the brain surface (outer panels) and shown on the sagittal slice $(x=3)$ (middle panel) (FWE, $p<0.05$ ). The white circles indicate the approximate location of the regions in which the parameter estimates were extracted in the peak voxel and gray and white matter adjusted volumes were calculated in $5 \mathrm{~mm}$ spheres around the peak. $\boldsymbol{B}$, Changes in brain structure and function with age are presented for the only region showing an effect of age on the SOSI-Color switch contrast, the right superior RLPFC. Left, Bar chart representing event-related RLFPC activation in SOSI switch and Color switch trials in the three age groups (mean \pm SE). Middle, Scatterplot of the BOLD response difference between SOSI switch and Color switch trials as a function of age. Right, Scatterplot of gray (gray circles) and white (black circles) matter adjusted volume as a function of age. The full lines represent significant correlations between functional or structural measures and age. The dashed line represents nonsignificant correlation with age.

Table 2. Results of multiple regression analyses performed to test whether the BOLD signal changes with age were a consequence of structural and performance differences

\begin{tabular}{lrrr}
\hline & $\beta$ & $t$ & $p$ \\
\hline$[-30,24,0]$ IFG SI-SO parameter estimate & & & \\
$R^{2}=0.220$ & & & \\
Gray matter adjusted volume IFG & 0.233 & 0.87 & 0.390 \\
White matter adjusted volume IFG & 0.253 & 1.13 & 0.267 \\
SI stay-S0 stay RT & -0.047 & -0.29 & 0.774 \\
SI stay-S0 stay accuracy & 0.106 & 0.66 & 0.512 \\
Age & -0.378 & -1.70 & 0.099 \\
{$[36,45,30]$ Superior RLPFC SOSI-Color switch parameter estimate } & & & \\
$R^{2}=0.135$ & & & \\
Gray matter adjusted volume RLPFC & -0.206 & -0.83 & 0.412 \\
White matter adjusted volume RLPFC & 0.038 & 0.21 & 0.832 \\
SOSI switch-Color switch RT & -0.030 & -0.15 & 0.880 \\
SOSI switch-Color switch accuracy & 0.075 & 0.42 & 0.681 \\
Age & -0.466 & -1.99 & 0.055 \\
\hline
\end{tabular}

Measures of performance, adjusted volumes of gray and white matter, and age were entered together. The results showed that the association between BOLD and age remained marginally significant when taking into account structural and performance parameters.

significantly decreased with age $(r=-0.318, p=0.055)$. VBM analyses revealed that gray matter volume in the right superior RLPFC decreased with age $(r=-0.682, p<0.001)$. White matter volume did not show significant changes with age $(p>$ 0.7 ) (Fig. 4B).

A regression analysis was performed with gray and white matter adjusted volumes and differences in accuracy and RT between SOSI and Color switch trials as regressors. Results indicated that age still marginally significantly accounted for BOLD response variance $(\beta=-0.466, p=0.055)$, while none of the structural or performance regressors showed significant effects (all $p>0.41$ ) (Table 2).

\section{Discussion}

This is the first study to combine a large scale behavioral study, and structural and functional imaging, to investigate the development of the selection and manipulation of self-generated thoughts, abilities that rely on RLPFC. The first aim of the study was to investigate the time course of performance changes from late childhood to adulthood on the alphabet task, which requires the flexible selection between $\mathrm{SO}$ and SI thoughts. Behavioral results revealed differences in the developmental trajectories of the different components of the task. Our second aim was to investigate the neural basis of the late-maturing components of the task. fMRI results revealed a decrease in activity with age as a continuous variable in the left IFG during the selection and manipulation of SI thoughts, and in the right superior RLPFC when switching between $\mathrm{SO}$ and SI thoughts. Our third aim was to evaluate the relationship between brain structure and function. The results demonstrated that changes in brain activation in this task do not entirely reflect local structural changes in gray and white matter volumes with age and may reflect the maturation of neurocognitive strategies. Previous developmental fMRI studies of executive function within the age range investigated here have mostly used WM and inhibition tasks. The alphabet task differs from WM tasks in two ways: (1) the representation that is manipulated has been selfgenerated; and (2) only one item of information has to be manipulated on each trial. This is the first time neurocognitive development has been investigated using a task that requires flexible selection between stimulus-oriented and self-generated thoughts.

In the behavioral study, participants aged 7-27 years performed a task requiring the flexible selection of SO or SI information, in the presence or absence of visual distractors. The different components of the alphabet task developed at different rates (Fig. 2). The ability to resist visual distractors exhibited the smallest changes with age, showing a small linear decrease in RTs $(\sim 130 \mathrm{~ms})$ and a small but prolonged quadratic decrease in accuracy $(\sim 7 \%)$, with adult performance reached by age 18.8 years (Fig. $2 B, D$ ). Studies of the interference of distracting perceptually derived information on the processing of task-relevant perceptually derived information also suggest limited developmental changes in the age range studied here (Adleman et al., 2002; Bunge et al., 2002; Booth et al., 2003; Schroeter et al., 2004), although WM load can amplify age differences (Olesen et al., 2007).

The ability to attend toward and manipulate SI (relative to $\mathrm{SO})$ thoughts exhibited a larger improvement in RT with age $(\sim 620 \mathrm{~ms})$. There was no change in accuracy, which indicates that participants of all ages were able to keep the letter sequence in mind appropriately (Fig. 2 D). By age 16, participants were able to perform the additional task demands in SI trials, i.e., attend to- 
ward and manipulate self-generated thoughts, as quickly as were adults. This development in the speed of manipulating selfgenerated thoughts may underlie improvements during adolescence in planning, reasoning and abstract thinking, abilities that rely on the manipulation of thoughts that are not directly derived from the environment (Anderson, 2001; De Luca et al., 2003; Rosso et al., 2004; Huizinga et al., 2006).

There was a large decrease in the RT cost of switching between selecting SI and SO thoughts (switch vs stay trials) with age ( $\sim 610 \mathrm{~ms}$ ), which followed a quadratic function flattening during late adolescence, with an inflection at 20 years. In adults, switching between tasks, in particular switching between two sets of stimulus-response mappings, is associated with slower responses and more errors immediately after a task switch (for review, see Monsell, 2003). Previous studies have shown improvement in task switching until mid-adolescence, with adult performance reached between 14 and 16 years (Reimers and Maylor, 2005; Crone et al., 2006; Davidson et al., 2006). Our results show a similar prolonged developmental change in performance when, rather than switching between stimulusresponse mappings, participants switch between using $\mathrm{SO}$ and SI information. Note that although switch trials could also be described as switches from the alphabet sequence (SO) to random letters (SI), or from no-distractor to distractor trials, it was precisely the ability to focus on self-generated thoughts despite the presence of SO information that was our focus of interest.

To investigate the recruitment of brain regions associated with the behavioral developmental changes observed, we performed a neuroimaging study using an adapted version of the alphabet task. The results revealed a core neural network in which activity in the majority of regions was not modulated by age. The comparison of SI vs SO blocks revealed activations in bilateral RLPFC, lateral PFC, ACC/preSMA, and parietal cortex. Fewer loci were activated in the SOSI switch vs Color switch contrast, with activations mostly located in the superior right RLPFC and the precuneus. The recruitment of RLPFC when switching between selecting SI and SO thoughts replicates earlier findings (Gilbert et al., 2005), while using a stricter comparison (Color switch). Our results suggest that by early adolescence the neural network involved in selecting and manipulating self-generated thoughts is recruited in a similar way as in adults (Gilbert et al., 2005, 2007, 2008). This is also the case for brain networks supporting response inhibition and WM (Luna et al., 2010).

Changes in BOLD response with age were investigated in the activation peaks from the main effects. Only two of these regions exhibited changes in activation with age. In the SI-SO contrast, the left IFG extending into the anterior insula showed a linear decrease in activation with age. This brain area is part of a network consistently recruited in functional imaging studies comparing two conditions differing in their need for cognitive control (Duncan, 2006), and shows a condition-specific decrease in activation with age in the flanker task (Bunge et al., 2002) and WM tasks (Scherf et al., 2006). In the SOSI switch-Color switch contrast, the right superior RLPFC exhibited a linear decrease in activation with age. Previous studies that have observed developmental changes in RLPFC have obtained inconsistent results. Decreases in RLPFC activation in adults compared with children (aged $\sim 8-12$ ) have been observed in response inhibition and response competition paradigms (Casey et al., 1997; Booth et al., 2003; Konrad et al., 2005), and during voluntary suppression of emotion (Lévesque et al., 2004). Increases in RLPFC activation have been observed in children compared with adults in a rela- tional reasoning task (Crone et al., 2009), and during childhood and adolescence in spatial WM tasks (Kwon et al., 2002; Schweinsburg et al., 2005; Olesen et al., 2007). These latter four studies involved the integration of visuospatial aspects of the stimuli, a requirement that was absent from the alphabet task or from other tasks that showed decreased RLPFC activation with age. Note that age differences in RLPFC activation time course have also been observed in fluid reasoning tasks, with delayed activations in children compared with adults (Wright et al., 2008; Crone et al., 2009). In addition, RLPFC activation has been found to be specific to the more difficult problems in adults but not in children (Crone et al., 2009). Resistance to distractors has been associated with a decrease in activation in the superior frontal sulcus between childhood and adulthood (Olesen et al., 2007); this result was not replicated here, possibly because of the low WM demands of the alphabet task.

A critical question this study aimed to address was whether age-related changes in brain activation could be accounted for by structural changes (Rivera et al., 2005). Gray and white matter volumes were evaluated in $5 \mathrm{~mm}$ spheres around the IFG and superior RLPFC peaks. Structural changes with age were observed in both regions. Gray matter volume, like brain activity, decreased with age, while white matter volume increased with age in IFG only. These results are consistent with the findings of increases in white matter volumes and decreases in gray matter volumes with age in the frontal cortex during adolescence (Giedd et al., 1999; Sowell et al., 1999, 2004; Barnea-Goraly et al., 2005; Shaw et al., 2008; Tamnes et al., 2010). Multiple regressions indicated that, for both the IFG and superior RLPFC, individual differences in gray and white matter volumes did not account for the observed decrease in activation with age. This suggests that the age-related functional changes are not purely consequences of structural maturation and may reflect the maturation of neurocognitive strategies. Consistent with these results, a recent study of visuospatial training in adolescent girls revealed that cortical thickness increases and brain activation changes after training did not occur in the same brain regions (Haier et al., 2009).

By combining behavioral, structural and functional data, this study shows that adolescence is associated with parallel changes in neural structure and activation, as well as performance, during the selection and manipulation of self-generated thoughts in the presence of perceptual distractors. These changes show independent associations with age, suggesting that adolescent cognitive development is characterized by a complex combination of behavioral and neural strategy changes and anatomical maturation.

\section{References}

Adleman NE, Menon V, Blasey CM, White CD, Warsofsky IS, Glover GH, Reiss AL (2002) A developmental fMRI study of the Stroop color-word task. Neuroimage 16:61-75.

Anderson V (2001) Assessing executive functions in children: biological, psychological, and developmental considerations. Pediatr Rehabil 4:119-136.

Ashburner J, Friston KJ (2000) Voxel-based morphometry-the methods. Neuroimage 11:805-821.

Barnea-Goraly N, Menon V, Eckert M, Tamm L, Bammer R, Karchemskiy A, Dant CC, Reiss AL (2005) White matter development during childhood and adolescence: a cross-sectional diffusion tensor imaging study. Cereb Cortex 15:1848-1854.

Blakemore SJ, Choudhury S (2006) Development of the adolescent brain: implications for executive function and social cognition. J Child Psychol Psychiatry 47:296-312.

Booth JR, Burman DD, Meyer JR, Lei Z, Trommer BL, Davenport ND, Li W, Parrish TB, Gitelman DR, Mesulam MM (2003) Neural development of selective attention and response inhibition. Neuroimage 20:737-751. 
Bunge SA, Dudukovic NM, Thomason ME, Vaidya CJ, Gabrieli JD (2002) Immature frontal lobe contributions to cognitive control in children: evidence from fMRI. Neuron 33:301-311.

Burgess PW, Simons JS, Dumontheil I, Gilbert SJ (2005) The gateway hypothesis of rostral prefrontal cortex (area 10) function. In: Measuring the mind: speed, control, and age (Duncan J, Phillips L, McLeod P, eds). Oxford: Oxford UP.

Burgess PW, Dumontheil I, Gilbert SJ (2007) The gateway hypothesis of rostral prefrontal cortex (area 10) function. Trends Cogn Sci 11:290-298.

Casey BJ, Trainor RJ, Orendi JL, Schubert AB, Nystrom LE, Giedd JN, Castellanos FX, Haxby JV, Noll DC, Cohen JD, Forman SD, Dahl RE, Rapoport JL (1997) A developmental functional MRI study of prefrontal activation during performance of a Go-No-Go task. J Cogn Neurosci 9:835-847.

Christoff K, Gabrieli JDE (2000) The frontopolar cortex and human cognition: evidence for a rostrocaudal hierarchical organization within the human prefrontal cortex. Psychobiology 28:168-186.

Crone EA, Wendelken C, Donohue S, van Leijenhorst L, Bunge SA (2006) Neurocognitive development of the ability to manipulate information in working memory. Proc Natl Acad Sci U S A 103:9315-9320.

Crone EA, Wendelken C, van Leijenhorst L, Honomichl RD, Christoff K, Bunge SA (2009) Neurocognitive development of relational reasoning. Dev Sci 12:55-66.

Davidson MC, Amso D, Anderson LC, Diamond A (2006) Development of cognitive control and executive functions from $4-13$ years: evidence from manipulations of memory, inhibition, and task switching. Neuropsychologia 44:2037-2078.

De Luca CR, Wood SJ, Anderson V, Buchanan JA, Proffitt TM, Mahony K, Pantelis C (2003) Normative data from the CANTAB I: development of executive function over the lifespan. J Clin Exp Neuropsychol 25: 242-254.

Dumontheil I, Burgess PW, Blakemore SJ (2008) Development of rostral prefrontal cortex and cognitive and behavioural disorders. Dev Med Child Neurol 50:168-181.

Duncan J (2006) EPS Mid-Career Award 2004: brain mechanisms of attention. Q J Exp Psychol 59:2-27.

Dunn LM, Dunn LM, Whetton C, Burley J (1997) The British picture vocabulary scale, Ed 2. Windsor: nfer-Nelson.

Friston KJ, Holmes AP, Poline JB, Grasby PJ, Williams SC, Frackowiak RS, Turner R (1995) Analysis of fMRI time-series revisited. Neuroimage 2:45-53.

Giedd JN, Blumenthal J, Jeffries NO, Castellanos FX, Liu H, Zijdenbos A, Paus T, Evans AC, Rapoport JL (1999) Brain development during childhood and adolescence: a longitudinal MRI study. Nat Neurosci 2:861-863.

Giedd JN, Clasen LS, Lenroot R, Greenstein D, Wallace GL, Ordaz S, Molloy EA, Blumenthal JD, Tossell JW, Stayer C, Samango-Sprouse CA, Shen D, Davatzikos C, Merke D, Chrousos GP (2006) Puberty-related influences on brain development. Mol Cell Endocrinol 254-255:154-162.

Gilbert SJ, Frith CD, Burgess PW (2005) Involvement of rostral prefrontal cortex in selection between stimulus-oriented and stimulus-independent thought. Eur J Neurosci 21:1423-1431.

Gilbert SJ, Williamson ID, Dumontheil I, Simons JS, Frith CD, Burgess PW (2007) Distinct regions of medial rostral prefrontal cortex supporting social and nonsocial functions. Soc Cogn Affect Neurosci 2:217-226.

Gilbert SJ, Bird G, Brindley R, Frith CD, Burgess PW (2008) Atypical recruitment of medial prefrontal cortex in autism spectrum disorders: an fMRI study of two executive function tasks. Neuropsychologia 46:22812291.

Haier RJ, Karama S, Leyba L, Jung RE (2009) MRI assessment of cortical thickness and functional activity changes in adolescent girls following three months of practice on a visual-spatial task. BMC Res Notes 2:174.

Huizinga M, Dolan CV, van der Molen MW (2006) Age-related change in executive function: developmental trends and a latent variable analysis. Neuropsychologia 44:2017-2036.

Konrad K, Neufang S, Thiel CM, Specht K, Hanisch C, Fan J, HerpertzDahlmann B, Fink GR (2005) Development of attentional networks: an fMRI study with children and adults. Neuroimage 28:429-439.

Kriegeskorte N, Simmons WK, Bellgowan PS, Baker CI (2009) Circular analysis in systems neuroscience: the dangers of double dipping. Nat Neurosci 12:535-540.
Kwon H, Reiss AL, Menon V (2002) Neural basis of protracted developmental changes in visuo-spatial working memory. Proc Natl Acad Sci U S A 99:13336-13341.

Lévesque J, Joanette Y, Mensour B, Beaudoin G, Leroux JM, Bourgouin P, Beauregard M (2004) Neural basis of emotional self-regulation in childhood. Neuroscience 129:361-369.

Liddle AR (2007) Information criteria for astrophysical model selection. MNRAS 377:L74-L78.

Lu LH, Dapretto M, O’Hare ED, Kan E, McCourt ST, Thompson PM, Toga AW, Bookheimer SY, Sowell ER (2009) Relationships between brain activation and brain structure in normally developing children. Cereb Cortex 19:2595-2604.

Luna B, Padmanabhan A, O'Hearn K (2010) What has fMRI told us about the development of cognitive control through adolescence? Brain Cogn 72:101-113.

Maldjian JA, Laurienti PJ, Kraft RA, Burdette JH (2003) An automated method for neuroanatomic and cytoarchitectonic atlas-based interrogation of fMRI data sets. Neuroimage 19:1233-1239.

Maldjian JA, Laurienti PJ, Burdette JH (2004) Precentral gyrus discrepancy in electronic versions of the Talairach Atlas. Neuroimage 21:450-455.

Monsell S (2003) Task switching. Trends Cogn Sci 7:134-140.

O’Brien LM, Ziegler DA, Deutsch CK, Kennedy DN, Goldstein JM, Seidman LJ, Hodge S, Makris N, Caviness V, Frazier JA, Herbert MR (2006) Adjustment for whole brain and cranial size in volumetric brain studies: a review of common adjustment factors and statistical methods. Harv Rev Psychiatry 14:141-151.

Olesen PJ, Nagy Z, Westerberg H, Klingberg T (2003) Combined analysis of DTI and fMRI data reveals a joint maturation of white and grey matter in a fronto-parietal network. Brain Res Cogn Brain Res 18:48-57.

Olesen PJ, Macoveanu J, Tegnér J, Klingberg T (2007) Brain activity related to working memory and distraction in children and adults. Cereb Cortex 17:1047-1054.

Reimers S, Maylor EA (2005) Task switching across the life span: effects of age on general and specific switch costs. Dev Psychol 41:661-671.

Rivera SM, Reiss AL, Eckert MA, Menon V (2005) Developmental changes in mental arithmetic: evidence for increased functional specialization in the left inferior parietal cortex. Cereb Cortex 15:1779-1790.

Rosso IM, Young AD, Femia LA, Yurgelun-Todd DA (2004) Cognitive and emotional components of frontal lobe functioning in childhood and adolescence. Ann N Y Acad Sci 1021:355-362.

Scherf KS, Sweeney JA, Luna B (2006) Brain basis of developmental change in visuospatial working memory. J Cogn Neurosci 18:1045-1058.

Schroeter ML, Zysset S, Wahl M, von Cramon DY (2004) Prefrontal activation due to Stroop interference increases during development-an eventrelated fNIRS study. Neuroimage 23:1317-1325.

Schwartz G (1978) Estimating the dimension of a model. Ann Statist $6: 461-464$.

Schweinsburg AD, Nagel BJ, Tapert SF (2005) FMRI reveals alteration of spatial working memory networks across adolescence. J Int Neuropsychol Soc 11:631-644.

Shaw P, Kabani NJ, Lerch JP, Eckstrand K, Lenroot R, Gogtay N, Greenstein D, Clasen L, Evans A, Rapoport JL, Giedd JN, Wise SP (2008) Neurodevelopmental trajectories of the human cerebral cortex. J Neurosci 28: 3586-3594.

Sowell ER, Thompson PM, Holmes CJ, Jernigan TL, Toga AW (1999) In vivo evidence for post-adolescent brain maturation in frontal and striatal regions. Nat Neurosci 2:859-861.

Sowell ER, Thompson PM, Leonard CM, Welcome SE, Kan E, Toga AW (2004) Longitudinal mapping of cortical thickness and brain growth in normal children. J Neurosci 24:8223-8231.

Tamnes CK, Ostby Y, Fjell AM, Westlye LT, Due-Tønnessen P, Walhovd KB (2010) Brain maturation in adolescence and young adulthood: regional age-related changes in cortical thickness and white matter volume and microstructure. Cereb Cortex 20:534-548.

Wechsler D (1999) Wechsler abbreviated scale of intelligence (WASI). San Antonio, TX: Harcourt Assessment.

Wright SB, Matlen BJ, Baym CL, Ferrer E, Bunge SA (2008) Neural correlates of fluid reasoning in children and adults. Front Hum Neurosci 1:8. 